\title{
Euthanasie
}

\section{La position de la Société Suisse de Médecine et de Soins Palliatifs (SSMSP)}

\section{F. Stiefel, H. Neuenschwander}

La position de la Société Suisse de Médecine et de Soins Palliatifs (SSMSP) dans le débat sur l'euthanasie se fonde sur un questionnaire distribué en novembre 2000 à tous les membres [1], et sur une discussion des résultats au sein du comité de la société. Le texte suivant a été approuvé à une large majorité des membres du comité de la SSMSP.

La SSMSP admet qu'il y a divers arguments pour et contre l'assistance médicale au suicide et l'euthanasie active directe (voir définition) [2].

Mais au-delà des arguments éthiques, juridiques et philosophiques, l'expérience du terrain montre que les désirs de mort chez les personnes gravement malades sont très souvent l'expression d'un accablement physique ou psychique, dû aux douleurs, à la dyspnée, à l'humeur dépressive ou à des conflits familiaux [3]. L'expérience clinique et des études scientifiques démontrent qu'avec des traitements spécialisés, les demandes d'euthanasie active directe ou la demande d'assistance au suicide, s'avèrent en général passagères $[4,5]$.

La SSMSP attire l'attention sur le fait qu'au cours de ces dernières décennies, les soins palliatifs (voir définition) ont fait de grands progrès dans la prise en charge des personnes gravement malades [6]. Cela a conduit dans divers pays, comme en Angleterre ou en Australie, au développement des soins palliatifs: divisions avec lits dans les hôpitaux de soins aigus, hospices, services ambulatoires, services de consultations intra et extra-hospitalières et soins palliatifs à domicile. En Suisse également les soins palliatifs se sont développés dans le courant de ces dernières années. Mais un état des lieux au niveau national [7] montre qu'en Suisse l'offre reste insuffisante et disparate entre cantons (voir "Soins Palliatifs en Suisse»). Il est aussi à noter que l'accès aux soins palliatifs se limite principalement aux patients atteints de cancer.

Les instances politiques comme le Conseil Fédéral [8] ou le Conseil National [9], les sociétés professionnelles comme l'Association Suisse des infirmières et des infirmiers (ASI) et la Fédération des médecins suisses (FMH) [10] ou l'Académie suisse des sciences médicales (ASSM) [11], ont souligné la nécessité de développer les soins palliatifs en Suisse. La SSMSP

Correspondance:

Dr Friedrich Stiefel, PD

CHUV

Service de Psychiatrie de liaison

CH-1011 Lausanne

E-mail: frederic.stiefel@inst. hospvd.ch juge donc inopportun d'ancrer dans la loi suisse l'assistance médicale au suicide, de même que l'euthanasie active directe, avant que chaque personne atteinte de maladie grave et évolutive n'ait accès, si nécessaire, à une médecine palliative soumise à un contrôle de qualité. Ces objectifs, les standards concernant le contrôle de qualité dans l'offre des soins cliniques [12] et les recommandations de formation en soins palliatifs [13], ont été adoptés lors d'une journée nationale de consensus le $1^{\text {er }}$ février 2001 (voir «Manifeste de Fribourg») [14].

Tant que l'accès aux soins palliatifs n'est pas assuré partout en Suisse, nous ne devrions pas favoriser un ancrage légal de l'assistance médicale au suicide ou de l'euthanasie active directe. Par contre la SSMSP souhaite que l'offre en soins palliatifs se développe et que la discussion publique sur le thème des soins en fin de vie ("End of Life Care») continue.

\section{Références}

1 Neuenschwander H, Bittel N und Stiefel F. Mot clé «Euthanasie»: comment se déterminent les membres de la Société Suisse de Médecine et de Soins Palliatifs dans cette question? Infokara 2001; Vol. 2 (traduction française: SSMSP).

2 Assistance au décès - Rapport du groupe de travail au Département fédéral de justice et police. Mars 1999.

3 Stiefel F. Sterbewunsch, Suizidgedanken, Suizid und aktive Euthanasie bei Krebskranken mit fortgeschrittenem Leiden. In: Mettner M (Hrsg.). Wie menschenwürdig sterben? Zürich: NZN Buchverlag AG; 2000. S. 29-36.

4 Owen C, Tennat C, Levi J, Jones M. Suicide and euthanasia: patient attitudes in the context of cancer. Psycho-Oncology 1992;1:79-88.

5 Guex P. La maladie terminale. Dans: Guex P (éd.). Psychologie et Cancer. Manuel de psycho-oncologie. Lausanne: Editions Payot; 1989. p. 131-51.

6 Neuenschwander H, Steiner N, Stiefel F, de Stoutz N, Humbert N, St.-Laurent-Gagnon T. Médecine palliative en un coup d'œil - Un manuel à l'intention du corps médical. Ligue suisse contre le cancer: 2000.

7 Raemy-Bass C, Lugon JP, Eggimann JC. Etat des lieux: Les soins palliatifs en Suisse 1999/2000. Ligue suisse contre le cancer et Société Suisse de Médecine et de Soins Palliatifs: 2001.

8 Une réglementation explicite de l'euthanasie passive et de l'euthanasie active indirecte est envisagée. Le Conseil fédéral adopte le rapport consécutif au postulat Ruffy. Communiqué du 5 juillet 2000.

9 Motion Rossini au Conseil national du 22 juin 2000: Financement des soins palliatifs.

10 Les soins dans la période terminale de la vie. Déclaration commune de l'Association suisse des infirmières et infirmiers (ASI) et de la Fédération des médecins suisses (FMH) Berne: 2001.

11 Directives médico-éthiques sur l'accompagnement médical des patients en fin de vie ou souffrant de troubles cérébraux extrêmes. Académie suisse des sciences médicales (ASSM). 1995.

12 Eychmüller S, Knipping C, Lang M, Mazzocato C, TschoppHafenbrack A, Zlatiev-Scocard N. Standards de qualité pour la Médecine et les Soins Palliatifs en Suisse. Société Suisse de Médecine et de Soins Palliatifs: 2001

13 Porchet F, Eychmüller S, Steiner N. Formation en soins palliatifs. Projet de recommandations nationales. Société Suisse de Médecine et de Soins Palliatifs: 2001.

14 Eychmüller S, Porchet F, Stiefel F, von Wyss M. Le Manifeste de Fribourg: Une stratégie nationale pour le développement des soins palliatifs en Suisse. Société Suisse de Médecine et de Soins Palliatifs: 2001 


\section{Définitions}

\section{Soins Palliatifs, définition}

La médecine et les soins palliatifs comprennent tous les traitements médicaux, les soins physiques, le soutien psychologique, social et spirituel, destinés aux malades souffrant d'une affection évolutive non guérissable. Son but est de soulager la souffrance, les symptômes et d'assurer le confort et la qualité de vie du malade et de ses proches (Société Suisse de Médecine et de Soins Palliatifs. Statuts 2000, art. 3).

\section{Soins Palliatifs, prestations}

Les soins palliatifs sont prodigués par une équipe interdisciplinaire composée de personnes formées et motivées pour s'occuper du confort de la personne malade et de ses proches. Aucun traitement ni médicament n'est administré dans le but de provoquer la mort. Aucune mesure n'est prise dans le but de prolonger la vie à tout prix. "A la découverte de la Médecine et des Soins Palliatifs», Société Suisse de Médecine et de Soins Palliatifs (éditrice), janvier 2001.

La Société Suisse de Médecine et de Soins Palliatifs (SSMSP)

La SSMSP réunit plus de 1000 membres de professions médicales et soignantes. Elle est politiquement et de confession neutre (statuts 2000, art. 6). Des informations supplémentaires peuvent être commandées auprès du président de la SSMSP: Dr F. Stiefel, PD, Service de Psychiatrie de Liaison, CHUV, 1011 Lausanne.

\section{Euthanasie passive}

Renoncement à des mesures de survie ou arrêt des mesures de survie. Cette forme d'euthanasie n'est pas expressément réglée par la loi, mais elle est considérée comme permise. "Assistance au décès" Rapport du groupe de travail au Département fédéral de justice et police. Mars 1999.

\section{Euthanasie active indirecte}

Traitement des symptômes en mettant en œuvre des moyens qui pourraient raccourcir la durée de la survie, mais sans intention de la raccourcir. L'euthanasie active indirecte n'est pas expressément réglée dans le code pénal actuel, mais considérée comme en principe admise. "Assistance au décès» - Rapport du groupe de travail au Département fédéral de justice et police. Mars 1999.

\section{Assistance médicale au suicide}

Suicide avec l'aide d'un médecin. L'assistance au suicide, en Suisse, n'est pas punissable, pour autant qu'un motif égoïste puisse être exclu (article $115 \mathrm{du}$ code pénal). L'assistance au suicide est pratiquée par Exit, mais "n'est pas un acte médical» selon les directives de l'Académie Suisse des Sciences Médicales (ASSM).

\section{Euthanasie active directe}

Homicide rapide et indolore d'une personne dans le but d'abréger sa souffrance. L'euthanasie active directe est punissable en droit pénal selon les articles 111, 113 et 114 du code pénal.

\section{Soins Palliatifs en Suisse}

Une étude nationale sur l'état des lieux des prestations en soins palliatifs, mandatée par la Ligue Suisse contre le Cancer et la Société Suisse de Médecine et de Soins Palliatifs, montre qu'en Suisse il existe encore de grandes différences entre cantons dans l'offre, qui se concentre surtout dans les centres urbains et qui est réservée surtout aux patients atteints d'un cancer. Pour les autres malades, les enfants et les personnes âgées, l'accessibilité reste insuffisante. Cette situation est aggravée par un manque d'offre de formation en soins palliatifs au niveau national et par un contrôle de qualité encore en voie de développement.

\section{Manifeste de Fribourg}

Le $1^{\text {er }}$ février 2001 à Fribourg, plus de 550 participants, de diverses professions de la santé, ont plébiscité un manifeste pour le développement des soins palliatifs. Dans ce manifeste, une liste de mesures impliquent la Confédération (intégration des soins palliatifs dans le cursus des études médicales et infirmières, ainsi dans le catalogue des prestations de la LAMal). D'autres mesures sont de la compétence des cantons (analyse des besoins régionaux et transformation de structures existantes en services de soins palliatifs hospitaliers et ambulatoires). Le "Manifeste de Fribourg", ainsi que le résumé de «l'état des lieux des Soins Palliatifs en Suisse" peuvent être commandés auprès du Président de la Société Suisse de Médecine et de Soins Palliatifs. 